\title{
Research on User Access Pattern Mining Based on Web Log
}

\author{
Wu Tingting1)
}

\begin{abstract}
Aiming at the field of e-commerce, this paper analyzes e-commerce user behavior based on the data characteristics of e-commerce back-end logs and constructs a user behavior mining model. On the basis of Web user behavior theory, it analyzes user behavior based on interactive content. Based on the background of big data, the traditional data mining algorithm is further optimized, which greatly improves the operating efficiency of the algorithm. At the same time, a distributed file storage structure is adopted to improve the fault tolerance of system data processing. This paper studies the advantages and disadvantages of collaborative filtering recommendation algorithms. The Web user behavior mining system constructed in this paper can conduct multi-dimensional and efficient mining. It helps e-commerce merchants and content providers to understand their users and achieve better commercial value through precise marketing and accurate recommendations, and complete the upgrade of data-driven services.
\end{abstract}

Keywords: E-Commerce, Big Data, Web Log Mining, User Behavior

\section{Introduction}

Since the popularization of the Internet, the number of online audiences has continued to increase. How to enable them to obtain more convenient and intelligent services, Web Mining [1] technology has emerged. Through user behavior collection systems, data processing platforms, and machine learning algorithms for mining data characteristics, we can actively display the content that users are most interested in and generate personalized services. The main application directions of Web log mining are: collecting user visit traces, mining user visit behavior patterns, optimizing website structure, and personalized information recommendation services. These application directions are derived from actual commercial needs, and because of their huge application value[2], they are also popular research directions in academia.

$\mathrm{Lu} \mathrm{Lina[3]} \mathrm{and} \mathrm{others} \mathrm{proposed} \mathrm{a} \mathrm{set} \mathrm{of} \mathrm{standardized} \mathrm{procedures} \mathrm{for} \mathrm{data} \mathrm{preprocessing.} \mathrm{The}$ source data is Web logs, site files, and user session files, and it uses data purification, user identification, session judgment, and path supplementation. Finally, you can discover the user's browsing mode and site usage, helping site managers optimize site structure and services.

Received(April 11, 2020), Review Result(1st: June 6, 2020, 2nd: July 22, 2020), Accepted(July 27, 2020)

1) (Professor) Shanxi University of Finance and Economics, Shanxi Province, China email: 3765287432@qq.com 
E. Cohen[4] proposed to develop a corresponding user behavior mining system based on user data. The process is as follows: through the client's proxy to collect the user's page access data such as browsing time, URL, clicks and requests, transfer through the proxy, and then send the data back to the server. Then build a mining system to mine log data. Bunchner et al.[5] can summarize the relationship between logs and data tables through the migration mode of user interests. Then use standard data mining algorithms to do the mapping of log data to relational tables in the database. At the same time, it can further save the time of log data preprocessing. For large data sets, it reduces the pressure of storage and calculation and also saves bandwidth loss caused by data transfer.

Under normal circumstances, when data mining algorithms process massive data sets in a big data platform, the approach taken is to break down tasks into smaller tasks. These tasks are distributed to different child nodes for calculation through the scheduling system. After the calculation of each node, the calculation results are summarized. This is how the data mining algorithm parallelizes the data processing in the big data platform. Luo[6][7] and others have studied many multi-task mode mining systems, which are mainly divided into two categories. One is a distributed mining system, such as the JAM system of Columbia University, the Quest of IBM, and Weka4WS of the Tailia team. The other is a parallel data mining method, the focus of research is on the data mining algorithm itself, and the design of the data mining algorithm or data processing in parallel. This has certain requirements on the algorithm itself, that is, there are parts that can be parallelized in the implementation of the algorithm itself, which can effectively improve the processing capacity of the algorithm itself. After the parallel processing research of data mining is combined with big data technology, data mining has achieved good results on large data sets.

Recommendation technologies can be divided into collaborative filtering, content-based, and hybrid recommendation, among which collaborative filtering recommendation technology is the most commonly used recommendation method in the field of recommendation systems. User-based collaborative filtering was proposed by Goldberg et al. in 1992 and applied to spam filtering[8]. The idea of this algorithm is to calculate the similarity between users (usually using Euclidean distance), and by finding and Users with similar interest preferences of the target user find users with similar interests to the target user, select items that the target user has not used from the item pool of similar users, and recommend items for the target user. In 2001, Sarwar et al.[9] proposed item-based collaborative filtering. It was first used on the Amazon e-commerce platform to recommend products to users[10], and then played an important role in multiple content distribution fields. Function[11], the idea of the algorithm is 
to calculate the similarity between items, and the association between items can be established according to the calculation results, and items are recommended based on the historical behavior of the target user and the similarity of the items.

This paper focuses on the research and analysis of the application of web log mining related technologies in user behavior mining in the big data environment, and the recommendation model after log mining. In-depth analysis of the format of the Web log and the network environment, pre-processing of the source data, and extracting effective user access information. The processed log file is used as the original material for user behavior mining. Through pattern mining, the general access patterns of users are obtained, and at the same time, further improvements are made to the imperfections of the traditional collaborative filtering recommendation system, and finally the prototype of the user behavior mining system is constructed.

\section{Related Research}

\subsection{Web User Behavior and Web Log Mining}

Web user behavior, it refers to the behavior of netizens on the Internet. It also refers to the behavior patterns of network users and their network activities. It is a broad definition[12]. At present, the number of Chinese Internet users is very large, and the population characteristics are also very diverse, so it is difficult to give an exact definition of online user behavior. Therefore, we usually classify the behavior of network users from the perspective of the network applications or content they visit. Through the analysis of user access content, we can classify online user behaviors into five major categories, including: content acquisition, online shopping behavior, social behavior, life service behavior, and entertainment behavior.

According to different research properties and research methods, network data mining is generally divided into the following three types: Web log mining (Web Usage Mining, also known as Web usage mining), Web content and structure mining. Data mining is the process of mining the above three types of information and discovering knowledge that is of interest or very valuable to users. [Table 1] makes a comprehensive comparison of the above three different types of Web data mining: 
[Table 1] Web Mining Classification

\begin{tabular}{|c|c|c|c|c|c|}
\hline $\begin{array}{c}\text { Web } \\
\text { mining }\end{array}$ & $\begin{array}{c}\text { Data } \\
\text { characteristics }\end{array}$ & $\begin{array}{c}\text { Content } \\
\text { carrier }\end{array}$ & Mining method & Application & $\begin{array}{c}\text { Representative } \\
\text { content }\end{array}$ \\
\hline $\begin{array}{c}\text { Log (used) } \\
\text { mining }\end{array}$ & $\begin{array}{c}\text { Interactive } \\
\text { behavior data } \\
\text { session } \\
\text { characteristics }\end{array}$ & $\begin{array}{c}\text { Server log } \\
\text { Browser } \\
\log \end{array}$ & $\begin{array}{c}\text { Statistical } \\
\text { analysis, } \\
\text { clustering, } \\
\text { classification, } \\
\text { association } \\
\text { rules }\end{array}$ & $\begin{array}{c}\text { Behavior analysis } \\
\text { Traffic analysis } \\
\text { Conversion rate } \\
\text { Recommendation }\end{array}$ & $\begin{array}{c}\text { Relationship } \\
\text { tables, charts }\end{array}$ \\
\hline $\begin{array}{c}\text { Content } \\
\text { mining }\end{array}$ & $\begin{array}{c}\text { Semi structured } \\
\text { Unstructured }\end{array}$ & $\begin{array}{c}\text { text, } \\
\text { hypertext }\end{array}$ & $\begin{array}{c}\text { classification, } \\
\text { text mining, }\end{array}$ & $\begin{array}{c}\text { personalized content } \\
\text { service, }\end{array}$ & $\begin{array}{c}\text { phrase, concept, } \\
\text { ontology, word } \\
\text { vector }\end{array}$ \\
\hline $\begin{array}{c}\text { Structure } \\
\text { mining }\end{array}$ & $\begin{array}{c}\text { Link structure } \\
\text { Hierarchy }\end{array}$ & link & $\begin{array}{c}\text { Classification, } \\
\text { clustering }\end{array}$ & $\begin{array}{c}\text { Site Analysis and } \\
\text { Optimization }\end{array}$ & Chart \\
\hline
\end{tabular}

The basic components of web log records mainly include: user ID, visited URL, date and time of behavior trigger (such as click, browse, feedback, etc.), detailed information of user request (can distinguish GET and POST, HTTP version number, HTTP Response information and browser information)[13]. Among them, what is used in this article includes the user's unique identification ID, the time of the request, the specific behavior triggered by the request, and the URL accessed. The main research scope of this paper is the field of e-commerce, and it also includes the content characteristics of e-commerce system, such as static data such as commodities and site modules, as well as user behavior on commodities and interaction data with the site. Web log mining is based on user access logs stored on the server side of the website. These access records contain traces of access left by users after a series of operations. The goal of Web log mining is to understand user access behavior patterns, find hidden rules and knowledge in log records, and strive to provide users with more accuracy through technical means such as classification models, clustering models, association analysis, path discovery, and recommendation models. Personalized service.

\subsection{Research on Data Mining Algorithms}

Clustering algorithm is a type of unsupervised learning. Supervised learning means that the training samples have been given the correct classification results before learning. The clustering algorithm is the process of discovering the unknown class of each sample, and then putting together multiple samples of the same potential class to form the same class. The following [Table 2] contains some common clustering methods. 
[Table 2] Common Clustering Methods

\begin{tabular}{|c|c|c|c|}
\hline $\begin{array}{c}\text { Clustering } \\
\text { method }\end{array}$ & Typical algorithm & Segmentation method & Features \\
\hline $\begin{array}{c}\text { Based on } \\
\text { partition }\end{array}$ & $\begin{array}{c}\text { K-MEANS } \\
\text { K-MEDOIDS } \\
\text { CLARANS }\end{array}$ & $\begin{array}{c}\text { Construct different partitions } \\
\text { and evaluate them according to } \\
\text { some criteria }\end{array}$ & $\begin{array}{c}\text { Fast processing speed } \\
\text { and easy to use }\end{array}$ \\
\hline $\begin{array}{c}\text { Based on } \\
\text { hierarchy }\end{array}$ & $\begin{array}{c}\text { DIANA } \\
\text { AGNES } \\
\text { BIRCH }\end{array}$ & $\begin{array}{c}\text { Use certain guidelines to create } \\
\text { a hierarchy of data (or object) } \\
\text { sets }\end{array}$ & $\begin{array}{c}\text { The clustering results } \\
\text { can be explained } \\
\text { visually by drawing a } \\
\text { dendrogram }\end{array}$ \\
\hline $\begin{array}{c}\text { CAMELEN } \\
\text { density }\end{array}$ & DBSCAN & OPTICS & Based on connectivity and \\
density function & $\begin{array}{c}\text { Clusters of arbitrary } \\
\text { shape can be found in } \\
\text { data samples with } \\
\text { "noise" }\end{array}$ \\
\hline $\begin{array}{c}\text { Fuzzy } \\
\text { clustering }\end{array}$ & FCM & Based on membership & $\begin{array}{c}\text { Different from hard } \\
\text { division, quantify the } \\
\text { fuzzy relationship } \\
\text { between samples }\end{array}$ \\
\hline
\end{tabular}

Sequence mining is the process of finding frequently occurring sequence combinations from a set of ordered data sequences, and is an extension of the association rule algorithm. The difference is that the results of sequential pattern mining and objects are ordered[14], which can potentially discover the temporal connections between transactions. Commonly used sequence mining algorithms include: Apriori All, GSP, PrefixSpan, SPADE, etc.[15]. [Table 3] shows several commonly used sequence mining algorithms.

[Table 3] Common Sequence Mining Algorithms

\begin{tabular}{|c|c|c|c|c|}
\hline \multirow{2}{*}{ Algorithm type } & \multicolumn{2}{|c|}{ Aprior-like algorithm } & \multicolumn{2}{|c|}{ Pattern growth algorithm } \\
\hline & ApriorAll & GSP & FreeSpan & PrefixSpan \\
\hline $\begin{array}{l}\text { Candidate sequence } \\
\text { generation tree structure }\end{array}$ & $\begin{array}{l}\text { True } \\
\text { Hash }\end{array}$ & $\begin{array}{l}\text { True } \\
\text { Hash }\end{array}$ & $\begin{array}{l}\text { False } \\
\text { Hash }\end{array}$ & $\begin{array}{l}\text { False } \\
\text { WAP }\end{array}$ \\
\hline Algorithm efficiency & low & $\begin{array}{l}\text { Compared with } \\
\text { the ApriorAll } \\
\text { algorithm, it is } \\
\text { improved by } 2 \text { to } \\
20 \text { times }\end{array}$ & Faster than Aprior & $\begin{array}{l}\text { Faster than } \\
\text { FreeSpan }\end{array}$ \\
\hline Database scan times & $\begin{array}{l}\text { Many } \\
\text { times }\end{array}$ & Many times & 3 & 2 \\
\hline Database segmentation & False & False & True & True \\
\hline
\end{tabular}

The recommendation algorithm uses some historical behavior data of the user and the attributes of the recommended item, combined with a specific algorithm process, to analyze and speculate on the items that the user may be interested in [16]. Commonly used recommendation 
algorithms include the following:

\subsubsection{Recommendation Algorithm Based on Collaborative Filtering}

The collaborative filtering algorithm has been introduced in the previous article, and it is generally divided into UserCF and ItemCF. The main working principle of UserCF is to recommend items to users that are of the same group as their hobbies and that the user has not purchased. An example is as follows: User A and User B are both Bao Moms, and A and $\mathrm{B}$ can be regarded as belonging to the same user group. When user $\mathrm{A}$ has a recommendation requirement, he can analyze B's shopping history and find out those that A has not purchased and recommend to $\mathrm{A}$. Based on the above basic principles, UserCF mainly includes the following two steps:

1. Find users who belong to the same interest group as the target user

2. Find items that the user likes and that the target user has not purchased and recommend to the target user.

The main working principle of ItemCF is to recommend items that are similar to the items that users liked before. An example is as follows: User A has previously bought item B "Murder on the Orient Express", the algorithm will recommend item C "No Survival" to you based on this behavior. The algorithm believes that item $B$ and item $C$ are very similar Degree is because most users who like item B also like item C. Based on the above basic principles, the ItemCF algorithm is mainly divided into two steps:

1. Calculate the similarity between all items;

2. According to the similarity of items and the historical behavior of users, the recommendation results are generated.

In order to find the nearest neighbors or users, commonly used methods to measure similarity include cosine value and Pearson correlation coefficient.

\subsubsection{Content-based Recommendation Algorithm}

Content-based recommendations usually analyze a series of historical behaviors of users, such as items that have been rated. Based on the feature modeling of the items that the user has scored, the recommendation is combined with the user's feature, and the user's feature is extracted from the user's interest. Based on the above basic principles, content-based recommendation is mainly divided into three steps:

1. Construct feature representations of items based on their attributes;

2. Construct user characteristics by learning user historical behavior; 


\subsection{Big Data Processing Framework Hadoop}

Low cost, high scalability, and high performance are the main reasons for the rapid growth of Hadoop distributed systems in academic and industrial fields. Hadoop reduces operating costs and energy consumption of computer resources by integrating high utilization of storage, network and computing resources, and a centralized computing platform[17]. In order to realize the effective storage of $\log$ data, this paper selects HDFS distributed storage for efficient reading and writing of data and extended storage. At the same time, the MapReduce parallel computing framework is used to realize the parallelization of the algorithm[18].

MapReduce is an easy-to-use parallel programming model. Based on the Hadoop platform, MapReduce can enable a task to be split into each node in the cluster, thereby achieving parallel processing of tasks, usually used for parallelizable tasks of large data sets. MapReduce is composed of a master server node (Job Tracker) and multiple slave task nodes (Task Tracker). This structure is also called a master-slave architecture. This is a scalable and elastic architecture that has been widely used. The master server node (Job Tracker) is responsible for the scheduling of all tasks (these tasks are allocated on the slave nodes) and is responsible for monitoring the execution status of each task server. When the task on the slave server node (Task Tracker) fails, the master server node (Job Tracker) transfers the task to another slave server node (Task Tracker).

The principle of parallel programming in MapReduce can be summarized as processing a bunch of messy data according to certain characteristics, and summarizing the process we want to solve, that is, the process of Map and Reduce. In the Map phase, the input of the Map function is a key-value pair. After processing by the Map function, the intermediate key-value pairs are generated, and then sorted by keys. With the same key value is merged, the generated key value pair is the output of the Map. After the Shuffle phase of MapReduce, the reduced phase is already summarized data.

In the Reduce phase, the Reduce function is used to receive the key-value pairs output from the Map phase. According to the user's requirements, the Reduce function gets a smaller result set after processing, and then outputs it to HDFS. Usually, Reduce produces only one or zero output. The architecture of MapReduce. As shown in [Fig. 1]. 


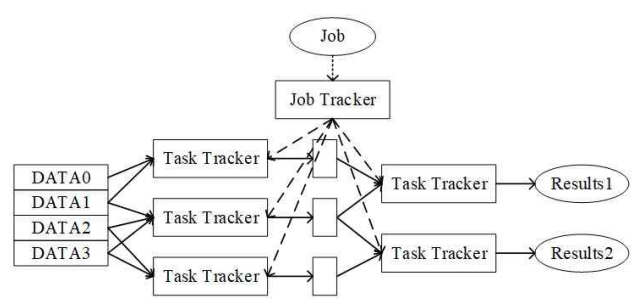

[Fig. 1] Map Reduce Architecture

HDFS (Distributed File System) can be used as the underlying distributed storage of multiple parallel computing frameworks, such as Spark and Hadoop's MapReduce. HDFS adopts a master-slave structure model consisting of a NameNode and multiple DataNodes. The master-slave structure of HDFS gives HDFS the advantage of high fault tolerance. As a distributed storage, it also has the characteristics of high throughput and high concurrency. It is very suitable for deployment on low-cost machines and can handle large-scale data sets., Has been widely used all the time. The slave node (DataNode) is used to perform computing tasks, is responsible for managing data storage and processing client read and write requests, and performs the tasks of NameNode creation, deletion, and copying of data blocks. HDFS divides a large data collection into several data blocks and stores them on multiple DataNodes. The storage structure is shown in [Fig. 2].

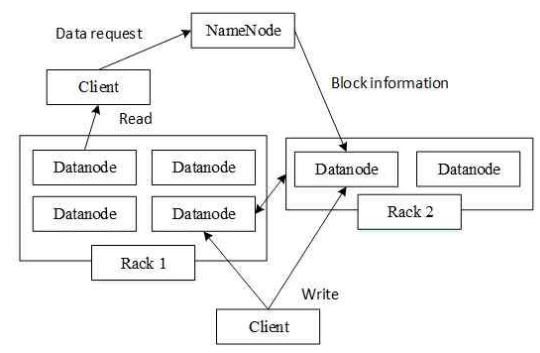

[Fig. 2] HDFS Data Storage Structure

In the HDFS file system, there is the most suitable file size, about $64 \mathrm{MB}$, which can make the scheduling of data files more efficient, and data backups between multiple machines at the same time make the entire system more reliable. Usually, the default number of copies is 3 copies. Using this strategy is a trade-off between minimizing read and write overhead and data reliability. Data files are scattered in different racks, and there are multiple copies of one data. This can minimize the network bandwidth overhead and read and write latency required by the Hadoop cluster during communication. When HDFS reads data, it determines which rack 
the read data request is closest to, and then requests the corresponding rack to obtain the data. If the HDFS cluster has multiple data centers in different regions, the copy of the local data center will be accessed first.

\section{Data Processing and User Behavior Recognition}

\subsection{Data Processing}

The data used in this article includes user purchase behavior records of a B2C e-commerce website A from April 2018 to December 2019 and Taobao user purchase behavior records from November 25, 2019 to December 3, 2019. The main data types of these two data sets are user information data, product information data, behavior data, and product category data. Each user, order, and product has its own independent ID. In order to prevent exposure of user privacy, it has been anonymized. The data is in UTF-8 encoding, and the time format is yyyy-MM-dd HH:mm:ss.

The pre-data of the unprocessed web log is rough, and there are some dirty data, such as repeated and wrong requests caused by network delays, noise data that has nothing to do with the subject, and some incomplete data. In addition to the normal visits of users, there are also search engines and crawlers, which will directly or indirectly affect the effect of data mining. Therefore, in order to make the original data meet the requirements of the experiment, we need to clean it.

For data cleaning, we first need to remove duplicate orders, products or users according to their unique ID numbers. The second is to clean missing and invalid values. This article uses Lagrangian interpolation to remove missing values. For some data, multiple key fields are missing and cannot be supplemented by other means as invalid values and can be removed directly. Then there is a unified format. Because there are many time representation formats, sometimes there is a problem of uniform format. Here, the time format is unified as yyyy-MM-dd HH:mm:ss. Finally, crawler data identification is required. According to the request IP, operation time interval, and access request header, most crawler requests can be identified, and this part of the data can be removed.

Data derivation is the creation of new features based on one or more features in the process of feature construction. You can make full use of the key field data. For example, the time when the user's order was created includes year, month, and day. At this time, we can derive this field to find the user's characteristics of each day and each month; the identification of 
Research on User Access Pattern Mining Based on Web Log

off-season months and peak-season months, etc.

\subsection{User Behavior Recognition}

User identification is the identification of the corresponding user in each log record, which is an important step in establishing a one-to-one or one-to-many relationship between the user and the log. Generally, it uses IP address, cookie identification, client information, registered user information, etc. to improve the accuracy of user identification. In practical applications, we need to take into account the user's privacy and the accuracy of user identification.

For session recognition, there are a variety of session recognition algorithms in the academic world, including: page access time threshold method, which is segmented by setting a single session duration threshold. Website homepage identification method, dynamic average time threshold method, etc. This article mainly uses the setting time threshold method, that is, when the total time of a single session does not exceed this time threshold.

Transaction recognition is to extract specific access patterns on the basis of session recognition, which is the basis of algorithms such as sequence mining and path recognition. The simplest method of transaction identification is the time window method, in which all pages that the user interacts with during a user session are divided into the length of a transaction time. The common identification method is the maximum forward sequence method. For example, the sequence generated by a user requesting a page during the browsing process is $\mathrm{ABCDE}$. Generally, there are multiple modules and pages in the system. The analysis and optimization of system sites are usually based on modules. Including site layout optimization, site nesting depth optimization, etc. User access to the marked page module will leave a record in the database as part of $\log$ data collection.

\section{Web User Access Pattern Mining System}

\subsection{Demand Analysis}

With the popularity of the Internet and more and more Web users, the amount of data on the Internet is increasing. On almost any website, such as e-commerce platforms such as Taobao and Jingdong; content distribution websites such as Baidu News and Toutiao. For audio and video websites such as Tencent Video and Douyin, as long as users visit, there will be corresponding recorded data. This will provide analysis data for the back-end engineering and 
technical personnel to complete the user's personalized service. This article mainly focuses on the e-commerce field. With the increase in the number of e-commerce platforms of the same nature, it is necessary to distinguish itself from the original marketing model and precision marketing. The transformation of the center's business model. According to the user's visit interest preferences, visit habits, visit paths and improved recommendation models, customized personalized services for target users are an important manifestation of Internet intelligence [19-22]. Starting from these modules, this chapter constructs the basic prototype of user access patterns[23][24].

\subsection{User Access Pattern Mining System}

The user's visit interest is the user's unique interest mode generated in the interaction between daily life and network information. For example, some users can quickly lock in a certain product and purchase it immediately, while some users will shop around before making further considerations. Some users like to watch different types of advertisements to reduce their choice costs[25][26]. This paper has completed the analysis of user interest and preference grouping. System users can select part of the user's access behavior. The background will convert the URL sequence and module tags generated by the user's access into a user access matrix model for processing. The clustering algorithm used in this paper combines fuzzy clustering and parallel processing of big data platforms, and adopts an improved parallelized fuzzy clustering algorithm. Through the model in the article, the user's visit interest can be converted into a visit feature matrix, and then the feature matrix can be clustered to divide users into corresponding interest groups. As shown in [Fig. 3], the optimal number of categories is determined through multiple experiments and comparisons as well as actual interpretability.

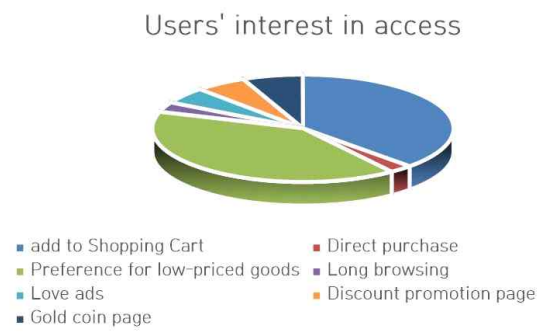

[Fig. 3] User Interest Classification

The user's access habits are related to the user's age, gender, occupation, and biological clock. For example, students mainly use the Internet on non-working days, while white-collar workers mainly focus on the time after get off work. This section completes the analysis of 
user habit classification, using the Xgboost algorithm. We select some users, because each person's attributes and laws are different, so different shopping habits are produced. According to the user's access distribution within a day, and the distribution on weekdays and weekends, the discretization of the time period can be performed to divide users into different categories, as shown in [Fig. 4].

User access habits

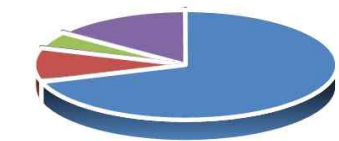

- Internet on weekdays (white collar)

" No work on weekends (Hi family)

- Late at night after get off work (night cats)

. Weekend (Otaku)

[Fig. 4] Mining User Access Habits

The site access analysis is divided into two parts. One is the site module distribution analysis, that is, whether users can use each module accurately and reasonably, and how attractive each module is to users. Through the module mark, can capture the user's access to each module. For a multi-module and multi-page system, we want to know the user's interest in certain modules, so as to adjust the distribution and functions of each module to achieve the purpose of site optimization. The second is the analysis of the user's access path. Through the user's access path, it can be seen whether the access nesting of the entire site is reasonable. For the more frequently accessed paths, we adjust the depth so that users can access them more conveniently, so as to optimize the site structure. The improved PrefixSpan algorithm on the Hadoop platform is used.

Comprehensive user recommendation is aimed at sparse user data, and when accurate recommendations are often not available, the migration learning method can assist the existing system in the historical data based on the vertical website or the old module. Recommended role. Connect users through shared product tags in the two fields to classify users. For the recommendation of our system users, we can recommend through the existing interest model, and at the same time construct a user feature matrix through the link of the tag, and use our scoring formula to score the same type of users. The final recommendation result is the recommendation generated by the transfer of the interest model of the source domain and the weighted recommendation of the interest model of the target domain.

The Web user access pattern mining system first analyzes the needs of the Web user access mining system to prove the value of the work done. Then analyze multiple modules of the 
system separately, carry out preliminary planning and realization, and finally form the preliminary prototype of the Web user mining system through the combination of modules.

\section{Conclusion}

As a practical research, web log mining has always been a hot direction of data mining, so it has great commercial and academic value. This paper first refers to a large number of literature and the actual needs of commercial companies, do a detailed research work, but also do a relatively adequate technical reserve. The whole process of Web log mining is analyzed one by one and studied deeply. At the same time, combined with the current needs of commercialization, the traditional web log mining system is improved, and the prototype of web user behavior mining system is completed, and each module and function are introduced in detail.

\section{References}

[1] Wang Fucong, Research and implementation of Web log mining system, China: Beijing University of Posts and Telecommunications, (2015)

[2] Li Zhongjun, Zhou Qihai, Shuai Qinghong, A recommendation system model based on isomorphic integration of content and collaborative filtering, Computer Science, (2009), Vol.36, No.12, pp.142-145.

[3] Lu Lina, Yang Tianling, Guan Xudong, Wei Hengyi, Research on data preprocessing in web log mining, Computer Engineering, (2000), Vol.26, No.4, pp.66-67.

[4] E. Cohen, B. Krishnamurthy, J. Rexford, Improving end-to-end performance of the web using server volumes and proxy filters, ACM SIGCOMM Computer Communication Review, (1998), Vol.28, No.4, pp.241-253.

[5] A. G. Bunchner, M. D. Mulvenna, Discovery Internet marketing intelligence through online analytical Web usage mining, ACM SIGMOD Record, (1998), Vol.27, No.4, pp.54-61.

[6] Luo P., Lu K., Shi Z. Z., Distributed data mining in grid computing environments, Future Generation Computer Systems, (2007), Vol.23, No.1, pp.84-91.

[7] Zhuang F. Z., He Q., Shi Z. Z., Multi-agent based on automatic evaluation system for classification algorithm, Proceedings of the International Conference on Information and Automation (ICIA'08), (2008), June 20-23; Zhangjiajie, China.

[8] Sarwar B., Karypis G., Konstan J., Item-based collaborative filtering recommendation algorithms, $10^{\text {th }}$ International Conference on World Wide Web, ACM, (2001), May 1-5; HongKong.

[9] Linden G., Smith B., York J., Amazon.com recommendations: item-to-item collaborative filtering, IEEE 
Internet Computing, (2003), Vol.7, No.1, pp.76-80.

[10] Xiang Liang, Recommendation system practice, China: People's Posts and Telecommunications Press, (2018)

[11] Liu Peng, Research on several issues of network user behavior analysis, China: Beijing University of Posts and Telecommunications, (2010)

[12] Huang Hongtao, Research on data preprocessing in web log mining, Heilongiiang Science and Technology Information, (2016), No.31, pp.195-196, DOI: 10.3969/j.issn.1673-1328.2014.31.214

[13] Wang Heqing, Web log mining and application based on sequential patterns, China: South China University of Technology, (2017)

[14] Yu Xiao, Ma Chuanxiang, Sequence pattern mining algorithm based on MapReduce, Application Research of Computers, (2015), Vol.32, No.11, pp.3312-3314, DOI: 10.3969/j.issn.1001-3695.2015.11.025

[15] Yao Ting, Research on personalized recommendation based on collaborative filtering algorithm, China: Beijing Institute of Technology, (2017)

[16] Wu Zhonghu, Research and application of collaborative filtering algorithm in Web recommendation system, China: East China Normal University, (2014)

[17] Liu Yongzeng, Zhang Xiaojing, Li Xianyi, Design of web log analysis system based on Hadoop/Hive, Journal of Guangxi University: Natural Science Edition, (2011), Vol.36, No.S1, pp.314-317.

[18] Zhou Ning, Xie Junyuan, Web service selection based on qualitative multi-user preferences, Chinese Journal of Electronics, (2011), Vol.39, No.4, pp.729-736.

[19] Wen Junjie, Qin Jia, Liu Ling, A Web service selection method under the condition of uncertain user preferences, Computer Application Research, (2010), Vol.27, No.6, pp.2147-2149.

[20] Liu Hongyan, Liu Zhenyu, E-Commerce Consumer Behavior Information Big Data Mining, International Journal of Database Theory and Application, (2016), Vol.9, No.7, pp.135-146.

[21] Akriti Gupta, Jyoteesh Malhotra, Research on a Dynamic E-commerce System based on Middleware Technology and Web Service, International Journal of Future Generation Communication and Networking, (2016), Vol.9, No.9, pp.203-214.

[22] Jiyoung Yoon, Soonhee Joung, A Consumer Recommendation System based on Big Data, International Journal of Smart Business and Technology, (2019), Vol.7, No.2. pp.25-30.

[23] Kokula Krishna Hari Kunasekaran, Research on E-commerce Customer Loyalty under Big Data, International Journal of Advanced Research in Big Data Management System, (2020), Vol.4, No.1, pp.1-16.

[24] Yan Bing, Yunyi Mo, The Relationship of Consumer Perceived Value, Online Word-of-Mouth and Behavioral Intention in Mobile E-commerce, International Journal of Smart Business and Technology, (2020), Vol.8, No.1, pp.13-20.

[25] Minwoo Park, Geunseong Jung, Kwanguk Kim, Hansung Kim, Jaehyuk Cha, A Framework for Evaluating Performance of Algorithms Extracting the Main Content from a Web Page, International Journal of Software Engineering and Its Applications, (2019), Vol.13, No.1, pp.17-24.

[26] Preeti Rathi, Geetam Singh Tomar, An Efficient Algorithm for Informational Retrieval using Web Usage Mining, International Journal of Hybrid Information Technology, (2019), Vol.12, No.2. pp.13-20. 